\title{
IR Bandwidth and Crystal Thickness Effects on THG Efficiency and Temporal Shaping of Quasi-rectangular UV pulses: Part II -Incident IR Intensity Ripple*
}

\author{
Paul R. Bolton and Cecile Limborg-Deprey, \\ Stanford Linear Accelerator Center, MS-18, \\ 2575 Sandhill Road, Menlo Park, California 94025.
}

\section{Introduction:}

We have investigated the effect of incident ir spectral bandwidth and crystal thickness, on uv pulses produced by third harmonic generation (THG) in a crystal pair. Our focus is on the third harmonic generation efficiency and longitudinal uv intensity profile parameters of SNLO predictions that are evaluated for three incident ir spectral bandwidths and a range of crystal thicknesses ${ }^{\mathbf{1}}$. These results represent a continuation of earlier work in which the effects of the same selected ir bandwidths and range of crystal thicknesses were investigated using a pair of BBO Type I crystals in a simplistic geometry for which the longitudinal intensity plateau has a zero slope, 'flattop' profile ${ }^{2}$. The current work is distinguished from the previous work by an imposed ripple on the ir intensity longitudinal profile and constitutes a portion of a Part II effort to which we have made reference ${ }^{2,3}$. As with preceding work, all third harmonic data are net results at the exit of the second BBO crystal.

Predictions are obtained with the modified SNLO code developed by Arlee Smith at the Sandia National Laboratories ${ }^{\mathbf{1}, \mathbf{4}}$. This modification has allowed us to pursue the 'coupled' case in which the output of the first BBO crystal is used as input to the second one. This includes both the fundamental and second harmonic light. Defined parameters are consistent with previous work. The presented cases are best results. The criteria for selection of these reported cases are highest THG efficiency combined with minimum intensity ripple in the plateau.

The incident ir pulse is quasi-rectangular with an imposed $5.2 \%$ (rms) intensity ripple added to the plateau. The ir pulse bandwidth is centered at $800 \mathrm{~nm}$. Second harmonic generation occurs in the first BBO crystal and THG occurs in the second crystal as a consequence of sum frequency generation. Type I phase matching is used throughout, so that for a negative uniaxial crystal ${ }^{5}$ :

$$
\begin{aligned}
& n_{2}^{e}(\theta)=n_{1}^{o} ;(S H G) \\
& 3 n_{3}^{e}(\theta)=2 n_{2}^{o}+n_{1}^{o} ;(T H G)
\end{aligned}
$$

\footnotetext{
* Work supported in part by the DOE Contract DE-AC02-76SF00515. This work was performed in support of the LCLS project at SLAC.
} 
where $n_{2}^{e}(\theta)$ and $n_{3}^{e}(\theta)$ are the angle dependent extraordinary refractive indices for the second and third harmonics respectively, and $n_{1}^{o}$ and $n_{2}^{o}$ are the ordinary refractive indices for the fundamental and second harmonic respectively.

Although our goal at this stage has not been to comply with all the LCLS injector laser specifications, the results provided here represent a parameter study that can be used to determine candidate bandwidth dependent, crystal thickness combinations for the detailed design of compliant THG subsystems. This simplistic geometry better elucidates acceptance bandwidth limitations that are intrinsic to the crystal material.

\section{Methodology:}

The basic geometry and plateau definition are as illustrated in figures 1 and 2 respectively of reference [2]. The two crystals separated by an ir half waveplate are all assumed to be lossless. The longitudinal profile of the incident ir pulse, which includes the $5.2 \%$ (rms) intensity ripple in the plateau, is shown in figure 1 . The other input ir pulse parameters are as follows:

$\begin{array}{lll}\text { spotsize, w (transverse FWHM) } & -3.4 \mathrm{~mm} & \text { (transverse Gaussian profile) } \\ \text { energy, } & -20 \mathrm{~mJ} & \\ \text { central wavelength, } & -800 \mathrm{~nm} \\ \text { rise and fall times, } & -0.69 \mathrm{psec} \\ \text { longitudinal FWHM } & -9.45 \mathrm{psec} \\ \text { longitudinal plateau duration } & -8.62 \mathrm{psec} \quad\left(\text { FWHM-0.6 } *\left(\tau_{\text {rise }}+\tau_{\text {fall }}\right) \text { ) }\right. \\ \text { peak intensity } & -12 \mathrm{GW} / \mathrm{cm}^{2} \\ \text { peak fluence } & -110 \mathrm{~mJ} / \mathrm{cm}^{2} \\ \text { intensity ripple (rms) } & -5.2 \% \quad \text { (longitudinal) }\end{array}$




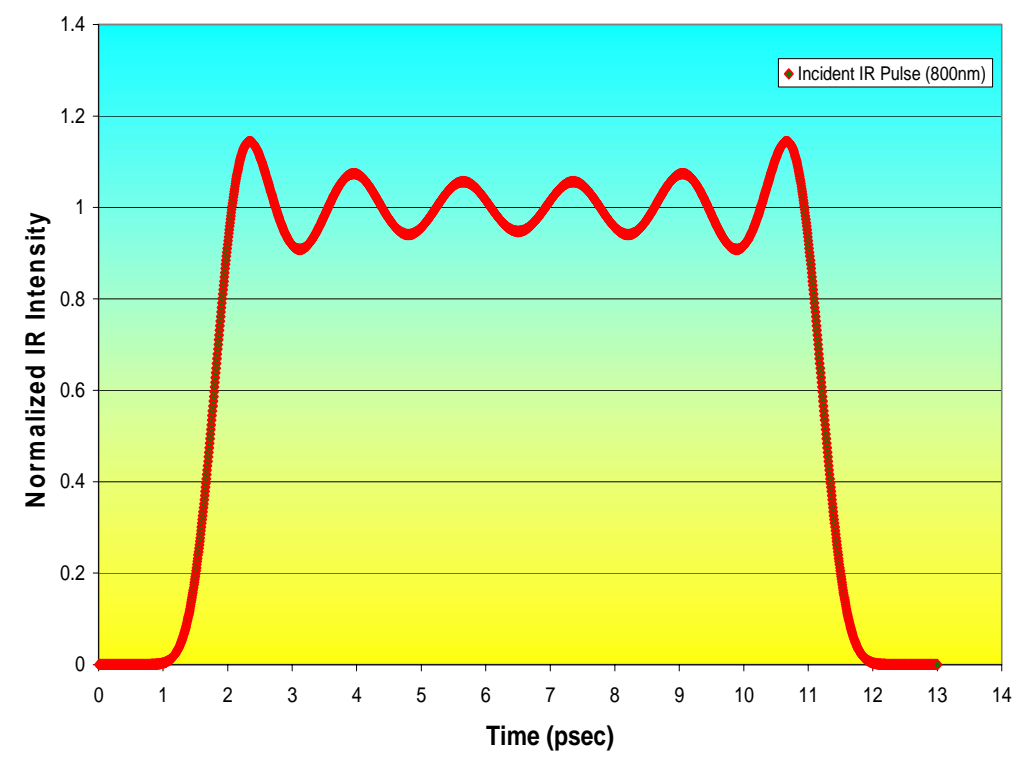

Figure 1. Normalized incident longitudinal ir intensity profile with plateau ripple.

There is no custom shaping of transverse ir profile and it is assumed to be Gaussian. The simple plane wave case is evaluated using the short pulse plane wave module of the SNLO code. Consequently, the incident ir spotsize is fixed and the SNLO predictions do not address focusing effects. Crystal parameters are as follows (as also specified in reference [2]):

$\begin{array}{ll}\text { phase matching } & - \text { type I } \\ \text { linear absorption coefficient } & -0.0015 \mathrm{~cm}^{-1} @ 800 \text { and } 400 \mathrm{~nm} \\ & 0.02 \mathrm{~cm}^{-1} @ 266.7 \mathrm{~nm} \\ \text { two-photon absorption coefficient } & -10^{-11} \mathrm{~cm} / \mathrm{W} \\ \text { nonlinear refractive index coefficient } & -8 \times 10^{-16} \mathrm{~cm}^{2} / \mathrm{W} \\ \text { reflectance per crystal surface } & -5 \%\end{array}$

Using the above input parameters we have examined the effect of crystal thicknesses for the three ir bandwidths: $2.5 \mathrm{~nm}(1.2 \mathrm{THz}), 5.0 \mathrm{~nm}(2.3 \mathrm{THz})$ and $10.0 \mathrm{~nm}$ (4.7 THz). A simple quadratic phase variation, as expressed below, is specified to set these bandwidths:

$$
\phi(t) \equiv-\omega_{o} t-\frac{b}{2} t^{2}
$$


Values of the chirp coefficient, b are: $0.754,1.485$ and $2.970 \mathrm{rad} / \mathrm{psec}^{2}$ for bandwidths 2.5, 5 and $10 \mathrm{~nm}$ respectively. There are no higher order phase variations. For all cases the incident ir longitudinal profile is as shown in figure 1. For a given incident ir bandwidth, the thicknesses of the first and second crystals are varied. For each thickness combination the THG efficiency, uv bandwidth and longitudinal (temporal) intensity profile parameters, as specified in the next section, are evaluated. Note that it is necessary to establish comparison with measured experimental results in order to obtain absolute efficiencies. The efficiency results reported here are to be interpreted relatively.

\section{Discussion of Results:}

Tables I, II, and III present best case results for the bandwidth and crystal thicknesses considered. These optimum cases are those for which the THG conversion efficiency is maximized and the plateau ripple is minimized. A detailed discussion of the crystal parameter choices (for example the estimated Type I longitudinal walkoff) can be found in section II of reference [2]. Optimum thicknesses for the first and second crystal do not exceed 0.8 and $0.3 \mathrm{~mm}$ respectively which is consistent the acceptance bandwidth for Type I phase matching. The following longitudinal intensity profile parameters are listed in each table for the uv wavelength:

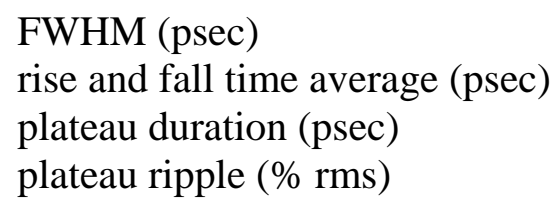

Note that the bandwidth (spectral FWHM) and net conversion efficiencies are given for the fundamental, second harmonic and third harmonic wavelengths (800, 400 and 266.7 $\mathrm{nm}$ respectively). What follows is a discussion of these tabulated results for each bandwidth case.

\section{III (a) - 10 nm Incident ir Case:}

Typical longitudinal intensity profiles exiting the first crystal are shown in figure 2. Because we continue to examine the 'coupled' case, these are relevant input profiles for THG. The incident $10 \mathrm{~nm}$ bandwidth is less than the intrinsic acceptance bandwidth of $18 \mathrm{~nm}$ for SHG (with $0.5 \mathrm{~mm}$ of $\mathrm{BBO}$ ) yet we anticipate some longitudinal pulse shaping for both the fundamental and the second harmonic. There is a clear central dip in the fundamental intensity profile that corresponds to a broad peak in the second harmonic intensity profile. With increased crystal thickness (greater than $0.5 \mathrm{~mm}$ ) this fundamental profile dip becomes more pronounced along with the central peak for the second harmonic. 


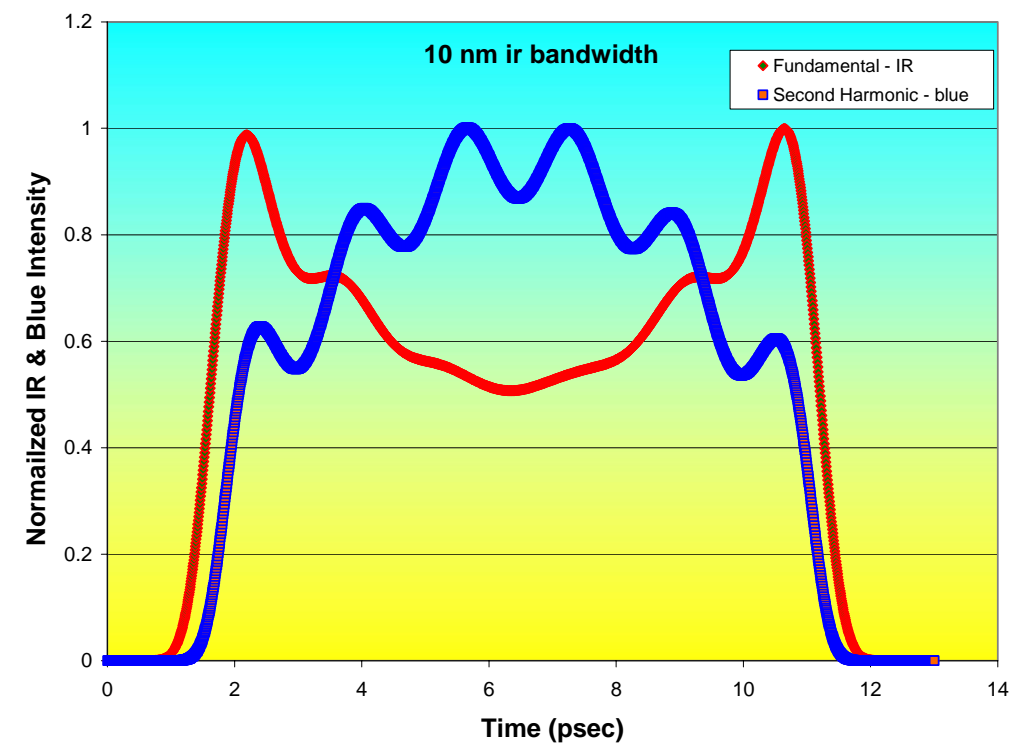

Figure 2. Normalized longitudinal intensity profiles of the fundamental and second harmonic pulses exiting the first BBO crystal of $0.5 \mathrm{~mm}(10 \mathrm{~nm}$ incident ir bandwidth).

Table I lists the results for the optimum cases using the incident $10 \mathrm{~nm}$ ir bandwidth. Figure 3 illustrates the best three of these cases. In general, regardless of the distinction between the fundamental and second harmonic profiles exiting the first crystal, it is seen that the longitudinal uv intensity profiles can qualitatively maintain the shape of the incident ir in figure 1. The two best tabulated cases are seen to be with a second crystal thickness of $0.1 \mathrm{~mm}$ and first crystal thicknesses of $0.5 \mathrm{~mm}$ (for which the uv conversion efficiency is the highest at $4.8 \%$ ) and $0.6 \mathrm{~mm}$ (for which the uv conversion efficiency is $4.4 \%$ ). The estimated acceptance bandwidth for a second crystal thickness of $0.1 \mathrm{~mm}$ is also $10 \mathrm{~nm}$. For a given crystal thickness combination, the three conversion efficiencies are comparable to those of the flattop case (agreement is within better than $10 \%$ in most cases) ${ }^{2}$. For these two cases in Table I, the intensity variations in the plateau are $8.3 \%$ and $7.2 \%$ for first crystal thicknesses of 0.5 and $0.6 \mathrm{~mm}$ respectively. These variations exceed the incident ir intensity plateau variation of $5.2 \%$. The tabulated uv intensity variations also significantly exceed those for the same crystal thicknesses in the flattop case ${ }^{2}$. With decreasing crystal thickness Table I shows that the rms intensity variation increases to $10.2 \%$ (twice the incident ir value) which is with a first crystal thickness of $0.4 \mathrm{~mm}$ and a second crystal thickness of $0.05 \mathrm{~mm}$. On the other hand, it will be seen for the narrowest incident ir bandwidth case of $2.5 \mathrm{~nm}$ for which uv conversion efficiencies are much higher (in Table III), that this intensity variation can be equal to or less than the incident ir value due to stronger saturation. 
Although the variation is not monotonic, Table I reveals a general decrease in plateau duration with increased crystal thicknesses as anticipated due to the reduced acceptance bandwidths of the pair of crystals. The plateau durations for the two best cases cited above are only 6.53 and 6.35 psec for first crystal thicknesses of 0.5 and 0.6 $\mathrm{mm}$ respectively. Plateaus durations are derived from the FWHM and the average of the rise and fall times. The FWHM is the more stable quantity, being less sensitive to rise and fall times. For most crystal thickness combinations, the FWHM values are within less than $10 \%$ of those for the flattop case ${ }^{2}$. Plateau variations are therefore mostly attributed to rise and fall time variations. Because of the imposed plateau ripple, rise and fall times can vary significantly (from 0.5 to 2.23 psec in Table I) and tend to be much lower than their counterparts in the flattop case ${ }^{2}$.

The uv spectral bandwidths for all the tabulated cases are at the $3 \mathrm{~nm}$ level which is within $10 \%$ of the ideal value of $3.3 \%$ (the ideal case being a sufficiently thin crystal for which the acceptance bandwidth significantly exceeds the incident ir value). For the thickest crystals ( $0.6 \mathrm{~mm}$ for the first crystal and $0.1 \mathrm{~mm}$ for the second one) we note the onset of bandwidth reduction below $3 \mathrm{~nm}$. For most crystal thickness combinations, the three bandwidth values (ir, blue and uv) agree to within better than $10 \%$ with those of the flattop case ${ }^{2}$.

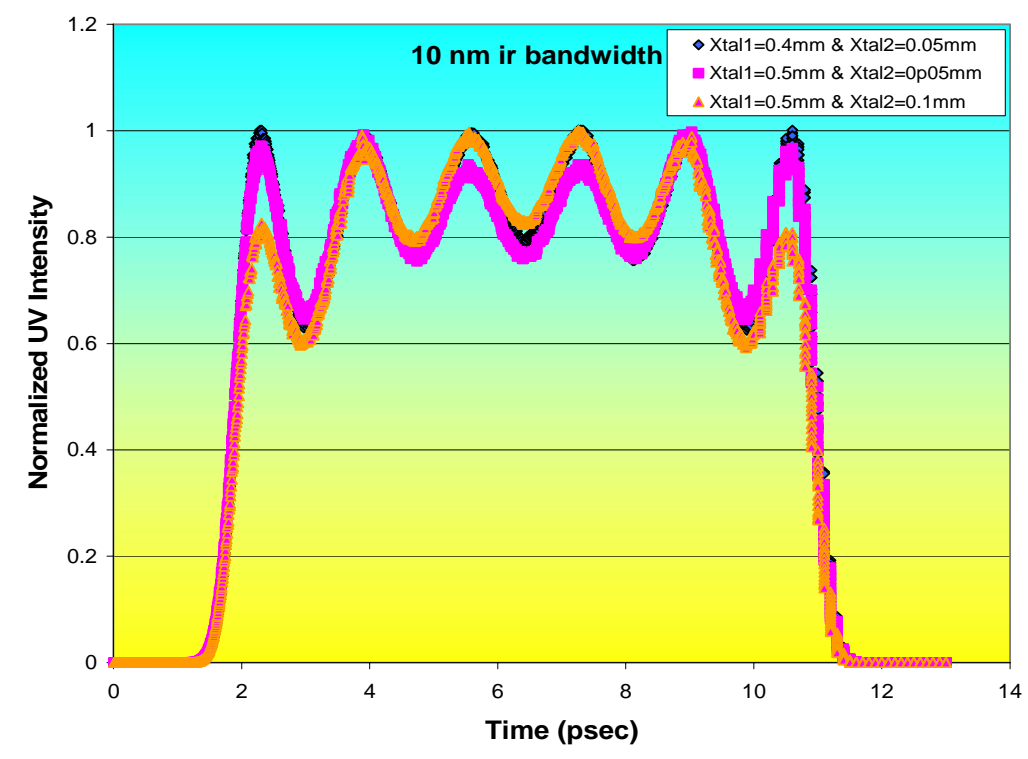

Figure 3. Normalized longitudinal uv intensity profiles exiting the second BBO crystal with a $10 \mathrm{~nm}$ incident ir bandwidth. 
Table I: $10 \mathrm{~nm}$ bandwidth Case

\begin{tabular}{|c|c|c|c|c|c|}
\hline $\begin{array}{l}\text { Crystal } 1 \text { depth (mm) } \\
\text { Crystal } 2 \text { depth (mm) }\end{array}$ & $\begin{array}{l}0.4 \\
0.05\end{array}$ & $\begin{array}{l}0.4 \\
0.1\end{array}$ & $\begin{array}{l}0.5 \\
0.05 \\
\end{array}$ & $\begin{array}{l}0.5 \\
0.1\end{array}$ & $\begin{array}{l}0.6 \\
0.1\end{array}$ \\
\hline $\begin{array}{l}\text { FWHM } \\
\text { (psec) }\end{array}$ & 9.10 & 8.93 & 9.10 & 8.94 & 8.77 \\
\hline $\begin{array}{l}\frac{\left(\tau_{\text {rise }}+\tau_{\text {fall }}\right)}{2} \\
\text { (psec) }\end{array}$ & 0.50 & 2.23 & 0.52 & 2.01 & 2.02 \\
\hline $\begin{array}{l}\text { Plateau } \\
\text { Duration } \\
\text { (psec) }\end{array}$ & 8.51 & 6.26 & 8.48 & 6.53 & 6.35 \\
\hline $\begin{array}{ll}\text { Bandwidth } & \text { ir } \\
(\mathrm{nm}) & \text { blue } \\
& \text { Uv }\end{array}$ & $\begin{array}{l}9.0^{*} \\
4.5 \\
3.0\end{array}$ & $\begin{array}{l}9.2^{*} \\
4.5 \\
3.0\end{array}$ & $\begin{array}{l}9.3^{*} \\
4.3 \\
3.0\end{array}$ & $\begin{array}{l}9.5^{*} \\
4.3 \\
3.0\end{array}$ & $\begin{array}{l}9.5^{*} \\
3.3 \\
2.9\end{array}$ \\
\hline $\begin{array}{l}\text { Plateau uv } \\
\text { Intensity } \\
\text { Variation } \\
\text { (rms - \% of peak) }\end{array}$ & 10.4 & 8.5 & 9.0 & 8.3 & 7.2 \\
\hline $\begin{array}{l}\text { Net } \quad \text { ir } \\
\text { Conversion blue } \\
\text { Efficiency (\%) uv }\end{array}$ & $\begin{array}{r}48.2 \\
31.5 \\
1.4\end{array}$ & $\begin{array}{r}47.0 \\
29.2 \\
4.8\end{array}$ & $\begin{array}{r}42.2 \\
37.4 \\
1.4\end{array}$ & $\begin{array}{r}41.0 \\
35.1 \\
4.8\end{array}$ & $\begin{array}{r}39.4 \\
37.0 \\
4.4\end{array}$ \\
\hline
\end{tabular}

* strong double peaked structure on ir spectra

\section{III (b) - 5 nm Incident ir Case:}

For the $5 \mathrm{~nm}$ incident ir bandwidth case, figure 4 reveals typical longitudinal intensity profiles for the fundamental and second harmonic light exiting the first crystal of thickness $0.6 \mathrm{~mm}$. While the central dip in the fundamental profile is clear, it is notably less than that for the $0.5 \mathrm{~mm}$ crystal in the $10 \mathrm{~nm}$ ir bandwidth case. Correspondingly the broad peak for the second harmonic is also reduced. We expect these results because the $5 \mathrm{~nm}$ bandwidth is significantly less than the estimated $15 \mathrm{~nm}$ acceptance bandwidth for Type I SHG.

Table II lists optimum results for the $5 \mathrm{~nm}$ bandwidth and the normalized longitudinal intensity profiles for the best three of these are illustrated in figure 5 . Relative to the $10 \mathrm{~nm}$ case (figure 3), the plateau intensity variation shown in figure 5 is 
reduced and the profiles are slightly more rectangular. The two best cases of Table II correspond to a first crystal thickness of $0.7 \mathrm{~mm}$ and second crystal thicknesses of 0.25 and $0.3 \mathrm{~mm}$ for which the net uv conversion efficiencies are $18.3 \%$ and $21.5 \%$ respectively. Once again, for a given crystal thickness combination, the three conversion efficiencies are comparable to those of the flattop case (agreement is within better than $10 \%$ in most cases) ${ }^{2}$. However, for the rippled plateau, efficiencies are about four times greater than the optimum ones for the $10 \mathrm{~nm}$ ir bandwidth and this is attributed mostly to the comparable THG acceptance bandwidth of 4.0 and $3.3 \mathrm{~nm}$ for second crystal thicknesses of 0.25 and $0.3 \mathrm{~mm}$ respectively. For these two cases in Table II, the rms intensity variation in the plateau is reduced to $6.8 \%(0.25 \mathrm{~mm}$ thickness) and $5.8 \%$ $(0.3 \mathrm{~mm}$ thickness) which still exceed the input ir variation of $5.2 \%$ and also exceed the uv intensity variation for the same crystal thicknesses in the flattop case ${ }^{2}$. With the exception of the thickest crystal case in Table II, the rms variation increases with decreasing thickness and this is attributed to the lower uv conversion efficiency. Acceptance bandwidths reduce with increasing crystal thickness which means that, although we observe higher uv conversion efficiencies with increased crystal thickness, we also generally anticipate reductions in plateau duration and slight reductions spectral bandwidth.

As with the $10 \mathrm{~nm}$ bandwidth case, for most crystal thickness combinations, the FWHM values agree with those for the flattop case (to within better than $10 \%{ }^{2}$. The plateau variation is again observed to be more sensitive to crystal thicknesses than the FWHM value due to the dependence on rise and fall times. For most crystal thickness combinations, the FWHM values are within less than $10 \%$ of those for the flattop case ${ }^{2}$. However, because of the imposed plateau ripple, rise and fall times can vary significantly (from 0.52 to $2.09 \mathrm{psec}$ in Table II) and tend to be much lower than their counterparts in the flattop case ${ }^{2}$.

Of the two best cases in Table II, the one with a $0.7 \mathrm{~mm}$ first crystal and a 0.25 $\mathrm{mm}$ second crystal has a notably higher plateau duration (lower rise and fall time) even though the uv conversion efficiency is about $15 \%$ lower and the rms intensity variation in the plateau is about $17 \%$ higher than for the $(0.7 \mathrm{~mm}+0.3 \mathrm{~mm})$ crystal pair. Also, the uv spectral bandwidth remains almost constant near $1.5 \mathrm{~nm}$, which is within $10 \%$ of the ideal thin crystal value of $1.7 \mathrm{~nm}$. Yet, for a given crystal thickness combination, all three bandwidth values are within $10 \%$ of the bandwidth values for the flattop case ${ }^{2}$. 


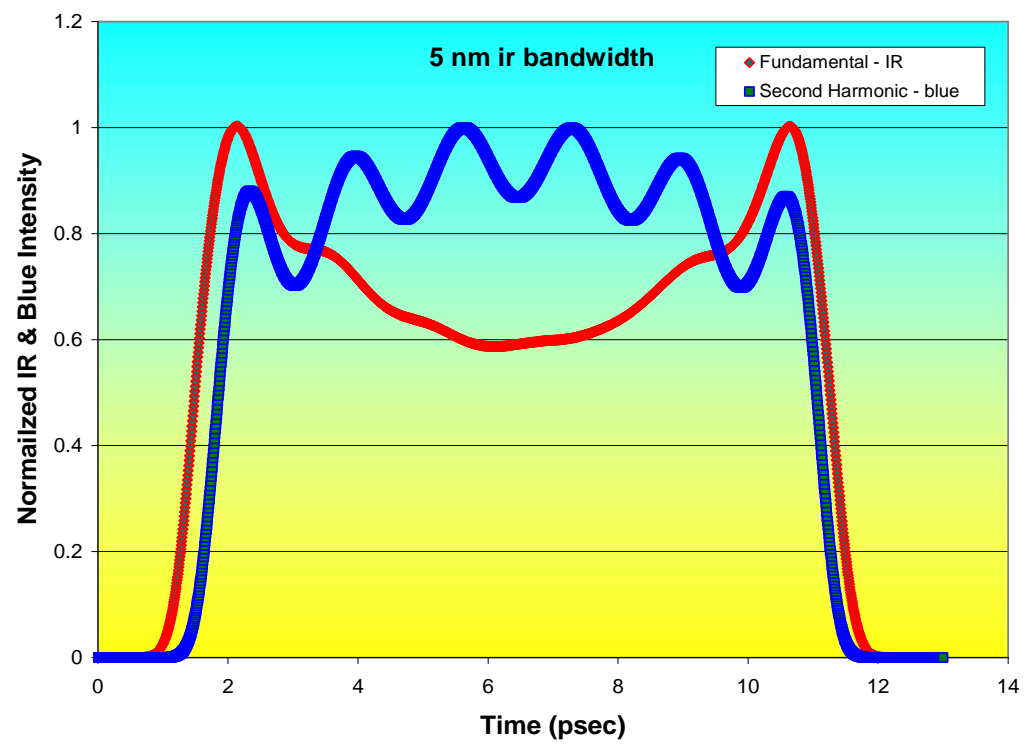

Figure 4. Normalized longitudinal intensity profiles of the fundamental and second harmonic pulses exiting the first BBO crystal of $0.6 \mathrm{~mm}$ (5 $\mathrm{nm}$ incident ir bandwidth). 
Table II: 5 nm bandwidth Case

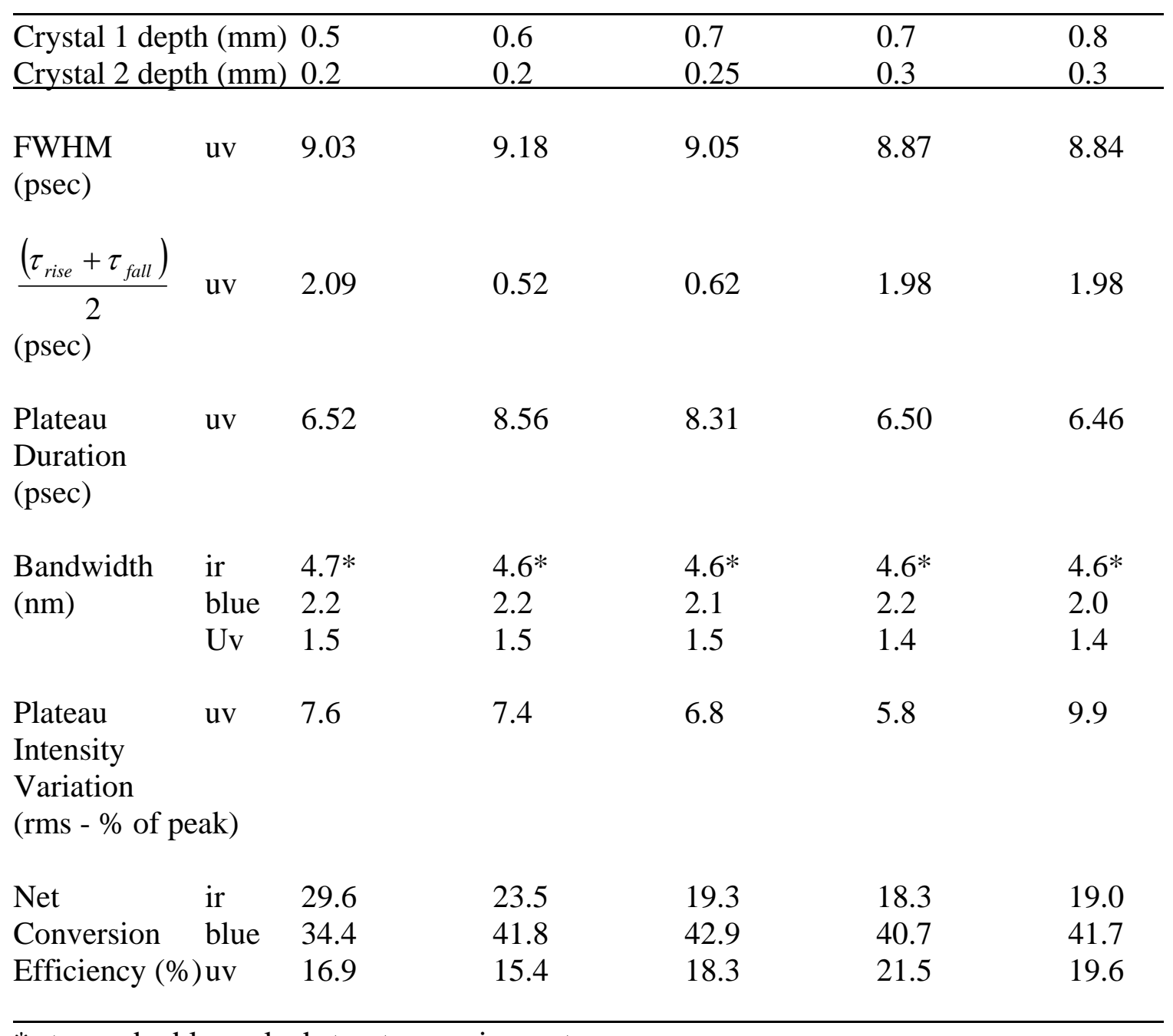

* strong double peaked structure on ir spectra 


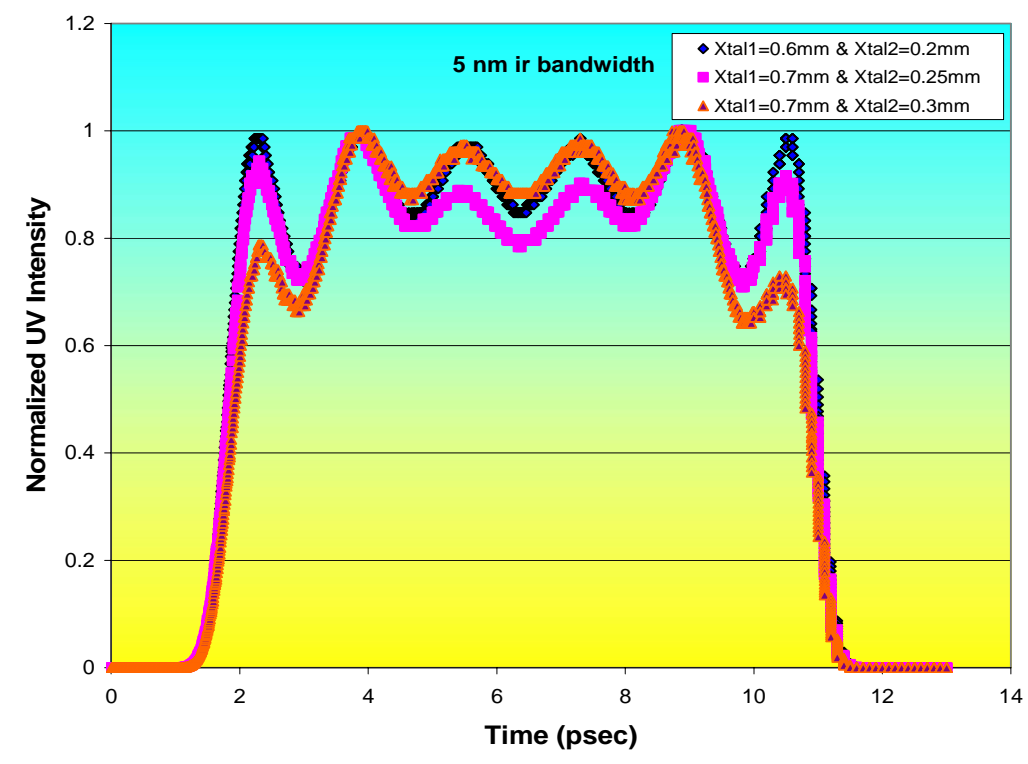

Figure 5. Normalized longitudinal uv intensity profiles exiting the second BBO crystal with a $5 \mathrm{~nm}$ incident ir bandwidth.

\section{III (c) - 2.5 nm Incident ir Case:}

For the $2.5 \mathrm{~nm}$ incident ir bandwidth, typical longitudinal intensity profiles are shown in figure 6 for the fundamental and second harmonic light exiting the first BBO crystal (of $0.7 \mathrm{~mm}$ thickness). Due to the lower bandwidth, the central dip in the fundamental profile and broad peak in the second harmonic profile are significantly reduced relative to the corresponding results shown in figures 2 and 4 (for incident ir bandwidths of 10 and $5 \mathrm{~nm}$ respectively).

Optimum results for the $2.5 \mathrm{~nm}$ bandwidth case are given in Table III. Normalized intensity profiles for the best three cases of Table III are illustrated in figure 7 where it is shown that, of the three bandwidth cases, the intensity ripple in the plateau (rms) is lowest (compare to figures 3 and 5 for 10 and $5 \mathrm{~nm}$ bandwidths respectively) because of the onset of saturation in the uv conversion efficiency. In fact, for the thickest crystal pair in Table III, the rms intensity ripple of $5.0 \%$ is less than the incident ir value of $5.2 \%$. However, this variation understandably remains higher than that for the same crystal thickness combination in the flattop case; even though this difference in intensity variation between the rippled and flattop cases is noticeably less than that for the 5 and 10 $\mathrm{nm}$ ir bandwidths (as little as $2.0 \%)^{2}$. 


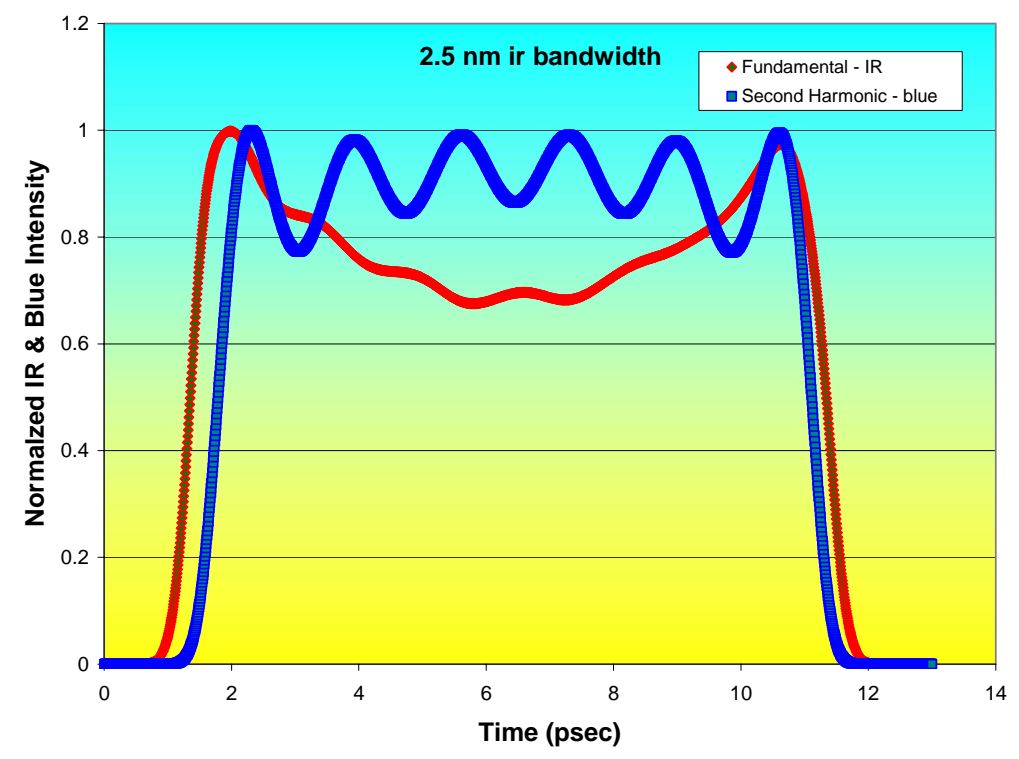

Figure 6. Normalized longitudinal intensity profiles of the fundamental and second harmonic pulses exiting the first BBO crystal of $0.7 \mathrm{~mm}$ (2.5 nm incident ir bandwidth).

Again, the plateau duration is observed to be more variable than the FWHM duration due to the rise and fall time variation with crystal thicknesses. For most crystal thickness combinations, the FWHM values are within less than $10 \%$ of those for the flattop case ${ }^{2}$. Due to the imposed plateau ripple, rise and fall times vary significantly (from 0.59 to 2.22 psec in Table III) and remain lower than their counterparts in the flattop case ${ }^{2}$.

The case where first crystal thickness is $0.7 \mathrm{~mm}$ and the second crystal thickness is $0.4 \mathrm{~mm}$ might be considered the best one of Table III. In this case the intensity ripple of $5.3 \%$ is very close to the incident ir level (5.2\%), the net uv conversion efficiency is high (32.1\%) and the high plateau duration of 8.69 psec is also near that of the incident ir pulse (8.62 psec). This combination of attributes is generally due to the higher uv conversion efficiency driven by this lowest ir bandwidth. Also, for a given crystal thickness combination, the three conversion efficiencies are comparable to those of the flattop case (agreement is within less than $10 \%$ in most cases) ${ }^{2}$.Table III also shows that, even for the thickest crystal combinations, the spectral bandwidths of the fundamental, second harmonic and third harmonic light exiting the second crystal are within about $10 \%$ of the ideal thin crystal values of $2.5,1.3$ and $0.8 \mathrm{~nm}$ respectively. 
Table III: 2.5 nm bandwidth Case

\begin{tabular}{|c|c|c|c|c|c|c|}
\hline \multicolumn{3}{|c|}{$\begin{array}{ll}\text { Crystal } 1 \text { depth (mm) } & 0.5 \\
\text { Crystal } 2 \text { depth (mm) } & 0.3 \\
\end{array}$} & $\begin{array}{l}0.6 \\
0.3\end{array}$ & $\begin{array}{l}0.6 \\
0.4\end{array}$ & $\begin{array}{l}0.7 \\
0.4\end{array}$ & $\begin{array}{l}0.8 \\
0.8\end{array}$ \\
\hline $\begin{array}{l}\text { FWHM } \\
\text { (psec) }\end{array}$ & uv & 9.19 & 9.25 & 9.08 & 9.26 & 9.22 \\
\hline $\begin{array}{l}\frac{\left(\tau_{\text {rise }}+\tau_{\text {fall }}\right)}{2} \\
\text { (psec) }\end{array}$ & uv & 0.61 & 0.59 & 2.22 & 0.65 & 1.35 \\
\hline $\begin{array}{l}\text { Plateau } \\
\text { Duration } \\
\text { (psec) }\end{array}$ & uv & 8.46 & 8.55 & 6.42 & 8.49 & 7.60 \\
\hline $\begin{array}{l}\text { Bandwidth } \\
\text { (nm) }\end{array}$ & $\begin{array}{l}\text { ir } \\
\text { blue } \\
\text { Uv }\end{array}$ & $\begin{array}{l}1.9^{*} \\
1.0 \\
0.7\end{array}$ & $\begin{array}{l}2.1^{*} \\
1.0 \\
0.7\end{array}$ & $\begin{array}{l}2.1^{*} \\
1.1 \\
0.7\end{array}$ & $\begin{array}{l}2.4^{*} \\
1.1 \\
0.7\end{array}$ & $\begin{array}{l}2.6^{*} \\
1.0 \\
0.7\end{array}$ \\
\hline $\begin{array}{l}\text { Plateau } \\
\text { Intensity } \\
\text { Variation } \\
\text { (rms - \% of }\end{array}$ & uv & 7.6 & 6.0 & 5.7 & 5.3 & 5.0 \\
\hline $\begin{array}{l}\text { Net } \\
\text { Conversion } \\
\text { Efficiency (9 }\end{array}$ & $\begin{array}{l}\text { ir } \\
\text { blue } \\
\text { uv }\end{array}$ & $\begin{array}{l}22.1 \\
26.0 \\
32.7\end{array}$ & $\begin{array}{l}15.0 \\
36.8 \\
29.0\end{array}$ & $\begin{array}{l}11.8 \\
30.5 \\
38.3\end{array}$ & $\begin{array}{r}8.1 \\
40.4 \\
32.1\end{array}$ & $\begin{array}{r}5.8 \\
45.8 \\
28.8\end{array}$ \\
\hline
\end{tabular}

* strong double peaked structure on ir spectra

These spectral bandwidth results are comparable to those for the flattop incident ir profile of the same crystal thicknesses (within $10 \%$ or less and see Table III of reference [2]). Furthermore, for the same crystal thickness combinations, the net conversion efficiencies for all three wavelengths are almost the same as those for the flattop case. 


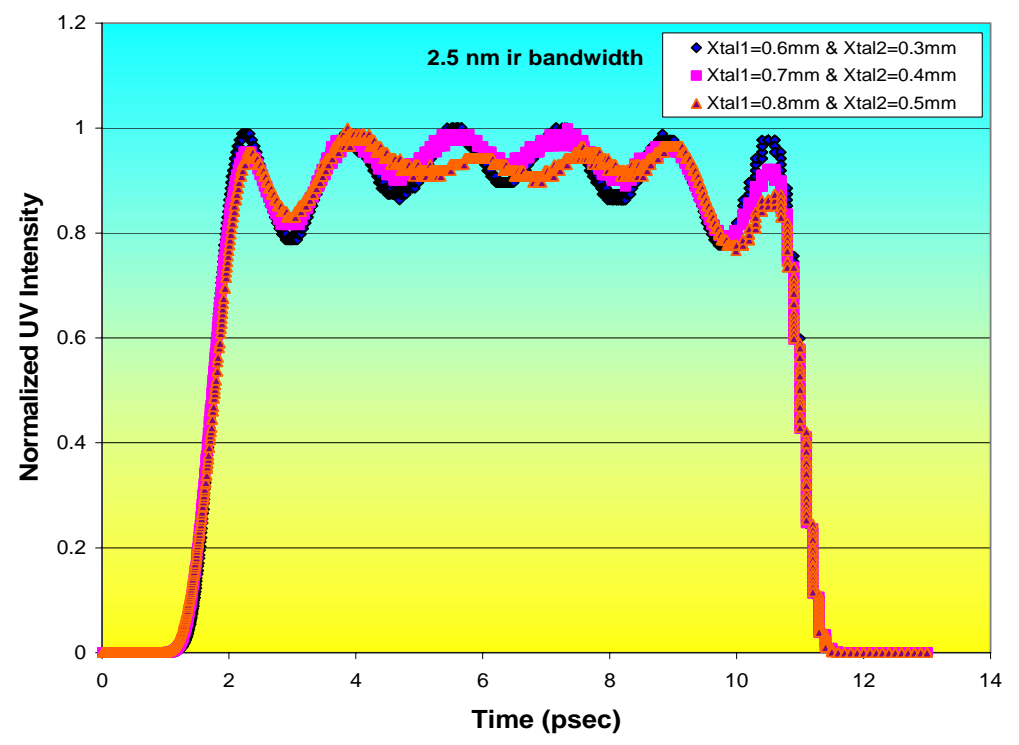

Figure 7. Normalized longitudinal uv intensity profiles exiting the second BBO crystal with a $2.5 \mathrm{~nm}$ incident ir bandwidth.

\section{Summary:}

It is generally true that, for a fixed combination of first and second crystal thicknesses, narrower incident ir bandwidths yield higher uv conversion efficiencies. Based on acceptance bandwidth arguments, this also means that for the narrower ir bandwidths we can use thicker crystal pairs while still preserving, to within some specified extent, the longitudinal intensity profile of the incident ir pulse (i.e. when constrained to recover to a specified extent the incident ir longitudinal intensity profile). Table IV below lists a summary of the important parameter ranges for the optimum cases that are given in Tables I, II and III. Most notable is the trend toward thicker crystals, higher uv conversion efficiencies, and lower intensity variation in the plateau for narrower incident ir bandwidths. 
Table IV: Summary for ir incident bandwidths 10, 5, and $2.5 \mathrm{~nm}$

Thickness Range (mm)

Crystal 1

Crystal 2

uv conversion efficiency (\%)

plateau duration (psec)

intensity variation

in plateau (\% rms)
Incident ir Bandwidth (nm)

\begin{tabular}{lcc}
\hline 10 & 5 & 2.5 \\
\hline $0.4-0.6$ & $0.5-0.8$ & $0.5-0.8$ \\
$0.05-0.1$ & $0.2-0.3$ & $0.3-0.5$
\end{tabular}

$1.4-4.8$

$15.4-21.5$

28.8-38.3

$6.35-8.51$

$6.46-8.56$

$6.42-8.55$

7.2-10.4

$5.8-9.9$

$5.0-7.6$

Table IV also indicates that upper limits to the thickness of the second crystal are imposed mainly by its acceptance bandwidth for THG. For the $2.5 \mathrm{~nm}$ bandwidth and with the parameter choices made in this work, it is possible to generate a longitudinal uv intensity profile that is very similar in duration and rms variation to that of the incident ir pulse. As we had also noted for the flattop case ${ }^{2}$, the $5 \mathrm{~nm}$ ir bandwidth may represent a reasonable compromise even though conversion efficiency is lower than for the $2.5 \mathrm{~nm}$ case and the rms intensity variation in the plateau is higher. It should also be noted that other parallel applications for an injector laser (that would employ parallel beamlines for example) can impose much higher ir bandwidth requirements and these are readily available commercially. It is to be emphasized here again that the uv conversion efficiencies are to be interpreted relatively. Absolute values can be inferred based on comparisons with experimental measurement.

As with the previous work ${ }^{2}$, there are natural extensions that include assessment of focusing effects, tests using intensity plateaus with nonzero slopes (with and without ripple), incorporating effects of transverse ir profile shaping, evaluating benefits of sequestering a portion of the incident ir for THG alone (i.e. an ir portion that bypasses the first crystal), investigation of other phase matching schemes, and the examination of shorter incident ir pulses using the same canonical bandwidths (in which higher incident ir intensities can result in higher uv conversion efficiencies). Using SNLO simulations we have set out to establish THG efficiencies and the relationships between specified incident ir pulse shapes and uv pulse shapes for different ir bandwidths and crystal thickness combinations. This catalogue of results can be consulted in the development of uv pulses with 'shaped' longitudinal intensity profiles that are produced by third harmonic generation. 


\section{References:}

1. SNLO nonlinear optics code available from A.V.Smith, Sandia National Laboratories, Albuquerque, NM 87185-1423.

2. P.R. Bolton and C. Limborg-Deprey, (SLAC) LCLS-TN-05-29 (Nov.'05).

3. C. Limborg-Deprey and P.R. Bolton, (SLAC) LCLS-TN-04-16 (Nov.'04) and SLAC-TN-05-012 (Nov.'04).

4. A.V. Smith, private communication.

5. “Nonlinear Optics”, second edition by R.W. Boyd, chapter 2, Academic Press 2003. 